\title{
Der Antisemitismus in Deutschland als Referenzrahmen: Transnationale Aspekte des Antisemitismus im Deutschschweizer Protestantismus
}

Ein Hauptmerkmal des sich im Deutschschweizer Protestantismus manifestierenden Antisemitismus war, dass für diesen das Deutsche Kaiserreich eine große Bedeutung als transnationaler Referenzrahmen besaß. ${ }^{1}$ Ein bedeutender Anteil der antisemitischen Äußerungen in den untersuchten Quellen nahm in irgendeiner Form Bezug auf Deutschland. Dieses Phänomen muss in einen größeren transnationalen Rahmen eingeordnet werden. Deutschland und vor allem der deutsche Protestantismus stellten für die Protestanten der Deutschschweiz zu jener Zeit ganz allgemein einen wichtigen Bezugspunkt dar. Germanophilie war in diesen Deutschschweizer Kreisen weit verbreitet, was sich nicht zuletzt an der Pfarrerschaft, den Kreisen der universitären Theologie und an den Redakteuren der protestantischen Periodika festmachen lässt. Die Verbundenheit war zum einen auf die konfessionelle Nähe zum Nachbarstaat, der von den Deutschschweizer Protestanten, wie die Schweiz selbst, als überwiegend,protestantischer Staat' verstanden wurde, zurückzuführen. Zum anderen war sie sprachlich-kulturell und biographisch bedingt, hatten doch viele Theologen der Deutschschweiz einen Teil ihrer Studienzeit im nördlichen Nachbarland verbracht und unterhielten wissenschaftliche Beziehungen zum Deutschen Reich. ${ }^{2}$ Bis Ende des Ersten Weltkrieges blieb das konfessionelle, sprachlich-kulturelle und oft auch theologische Verbundenheitsgefühl mit Deutschland im Deutschschweizer Protestantismus sehr ausgeprägt.

1 Die hier präsentierten Aspekte zur transnationalen Dimension des Antisemitismus im Protestantismus der Deutschschweiz basieren auf Forschungen für meine an der Universität Freiburg in der Schweiz verfasste Dissertation Antisemitismus im Deutschschweizer Protestantismus von 1870 bis 1950, die im Frühjahr 2017 im Berliner Metropol Verlag als Band 12 der Reihe Studien zum Antisemitismus in Europa erschienen ist.

2 Zur Germanophilie in der Deutschschweiz siehe: Klaus Urner, Die Deutschen in der Schweiz. Von den Anfängen der Kolonienbildung bis zum Ausbruch des Ersten Weltkrieges, Stuttgart 1976, v. a. S. 49-91. Die stark germanophilen Tendenzen im Deutschschweizer Protestantismus hatten sich etwa während des Deutsch-Französischen Krieges von 1870/71 gezeigt. Siehe exemplarisch: Die Stimme Gottes in dem Donner der Schlachten, in: Appenzeller Sonntagsblatt 9/35 (1870), Beilage S. 1-4. 
Dieser Beitrag analysiert die verschiedenen Dimensionen der Bedeutung des Deutschen Kaiserreichs als transnationaler Referenzrahmen für den Antisemitismus im Deutschschweizer Protestantismus. Die Untersuchungen basieren dabei auf einer Analyse der bedeutendsten protestantischen Zeitschriften der Deutschschweiz. ${ }^{3}$ Im zeitlichen Fokus steht das letzte Drittel des 19. Jahrhunderts, situativ erstreckt sich die Analyse jedoch über diesen Zeitrahmen hinaus. Die Zeitschriftenlandschaft war geprägt von einer Aufspaltung in verschiedene theologisch-kirchenpolitische Richtungen. Sie bildeten das rigide sogenannte Richtungswesen nach, das für den damaligen Protestantismus der Deutschschweiz charakteristisch war. Dem liberalen oder, freisinnigen' Protestantismus, der sich in einer Mehrheitssituation befand, stand die sich in verschiedene Unterströmungen gliedernde Richtung der als ,positiv' bezeichneten Konservativen gegenüber. Gerade die von Basel ausgehende Tradition des Pietismus spielte darin eine wichtige Rolle. Die Mittepartei der sogenannten Vermittler war theologisch nicht ohne Einfluss, aber zahlenmäßig klein. ${ }^{4} \mathrm{Ab}$ den $1830 \mathrm{er}$ Jahren hatte sich der Gegensatz zwischen konservativer und liberaler Theologie verschärft. Die Liberalen votierten für die Aufhebung des Bekenntniszwangs und eine sich an wissenschaftlich-kritischen Maßstäben orientierende Theologie, die etwa die Bibel als historisch gewachsenes Dokument erachtete. Beides waren Standpunkte, die in den Augen der biblizistisch geprägten Konservativen an den Grundfesten des Glaubens rüttelten. Die Bibel stellte für sie göttliche Offenbarung dar, und sie plädierten für die Beibehaltung des Bekenntniszwanges. ${ }^{5}$

Das damals den schweizerischen ähnlich wie den deutschen Protestantismus prägende Richtungswesen bildete sich ebenso in den antisemitischen Einstel-

3 Folgende Periodika sind von besonderer Bedeutung für diesen Beitrag. Konservativ-protestantisch: Appenzeller Sonntagsblatt, Christlicher Volksbote, Christlicher Volksfreund, Kirchenfreund, Der Freund Israels, Evangelisches Wochenblatt; liberal: Basler (Schweizerisches) Protestantenblatt, Reformblätter aus der bernischen Kirche/Schweizerische Reformblätter, Religiöses Volksblatt; ,vermittlerisch': Volksblatt für die reformi(e)rte Kirche der Schweiz. In der Schweiz hatte sich, im Gegensatz zum Deutschen Kaiserreich, kein protestantisches Zeitungswesen herausgebildet. Ausnahmen stellten in Ansätzen die Allgemeine Schweizer Zeitung sowie die Berner Volkszeitung dar.

4 Für das protestantische Richtungswesen siehe: Peter Aerne, Religiöse Sozialisten, Jungreformierte und Feldprediger. Konfrontationen im Schweizer Protestantismus 1920-1950, Zürich 2006, S. 27-75; Rudolf Gebhard, Umstrittene Bekenntnisfreiheit. Der Apostolikumstreit in den Reformierten Kirchen der Deutschschweiz im 19. Jahrhundert, Zürich 2003; Lukas Vischer/Lukas Schenker/Rudolf Dellsperger (Hg.), Ökumenische Kirchengeschichte der Schweiz, Freiburg Basel 1994, S. 218-240; Paul Schweizer, Freisinnig - Positiv - Religiössozial. Ein Beitrag zur Geschichte der Richtungen im Schweizerischen Protestantismus (Basler Studien zur historischen und systematischen Theologie, 18), Zürich 1972.

5 Einen Überblick über die theologischen Differenzen bietet: Schweizer, Freisinnig - Positiv Religiössozial (wie Anm. 4); Arnd Wiedmann, Imperialismus - Militarismus - Sozialismus. Der deutschschweizerische Protestantismus in seinen Zeitschriften und die großen Fragen der Zeit 1900-1930 (Geist und Werk der Zeiten, 83), Bern 1995, zusammenfassend S. 46-47. 
lungen ab. Diese waren im letzten Drittel des 19. Jahrhunderts unter den ,Positiven' und ,Vermittlern' wesentlich stärker verbreitet als unter den Liberalen. Zugleich gab es auch inhaltliche Differenzen zwischen den Richtungen. ${ }^{6}$ Auch was die Bedeutung des Deutschen Kaiserreichs als Referenzrahmen in Sachen Antisemitismus angeht, war diese für den konservativen und ,vermittlerischen ‘ Protestantismus in jenen Jahren weit größer als für den liberalen. Die Rezeption des Antisemitismus in Deutschland, insbesondere im deutschen Protestantismus, bot den Glaubensbrüdern (und -schwestern) in der Deutschschweiz insgesamt inhaltliche Orientierung in Sachen Judenfeindschaft. Daher übertrug sich die im Kaiserreich seit Mitte der 1870er-Jahre aufkommende antisemitische Welle ebenfalls auf den Deutschschweizer Protestantismus. ${ }^{7}$ In diesem Prozess lassen sich die nachfolgenden drei Dimensionen herausarbeiten, die sich im Einzelfall oft miteinander verschränkt zeigten.

\section{Antisemitismusrezeption als Katalysator für die Popularisierung der Judenfeindschaft}

Die Rezeption des Antisemitismus in Deutschland wirkte sich katalytisch auf die Popularisierung der Judenfeindschaft im Deutschschweizer Protestantismus aus. Wie Entwicklungen im deutschen Protestantismus in den protestantischen Periodika der Deutschschweiz im Allgemeinen auf ein reges Interesse stießen, so wandten sich die Zeitungen auch dessen antisemitischen Aktivitäten aufmerksam zu. Besonders ausgeprägt war dieses Interesse von konservativ-protestantischer Seite. Waren in den 1860er und in den frühen 1870er-Jahren umfangreichere antisemitische Artikel in den untersuchten theologischen und erbaulichen Zeitschriften noch eine Seltenheit, so änderte sich dies gegen Ende des Jahrzehnts. Mit dieser quantitativen Veränderung einher ging eine Gewichts-

6 Differenzen zeigten sich nicht zuletzt in antimodernistisch oder heilsgeschichtlich geprägten antisemitischen Diskursen.

7 Siehe aus der Fülle der Forschungen zum Anschwellen des Antisemitismus im ersten Jahrzehnt des Deutschen Kaiserreichs: Werner Bergmann/Ulrich Wyrwa, Antisemitismus in Zentraleuropa. Deutschland, Österreich und die Schweiz vom 18. Jahrhundert bis zur Gegenwart, Darmstadt 2011; Massimo Ferrari Zumbini, Die Wurzeln des Bösen. Gründerjahre des Antisemitismus. Von der Bismarckzeit zu Hitler (Das Abendland - N. F., 32), Frankfurt a. M. 2003; Werner Jochmann, Antisemitismus im Deutschen Kaiserreich 1871-1914, in: Derselbe, Gesellschaftskrise und Judenfeindschaft in Deutschland 1870-1945 (Hamburger Beiträge zur Sozial- und Zeitgeschichte, 23), Hamburg 1988, S. 30-98. Mit Blick auf den Protestantismus und Katholizismus: Wolfgang E. Heinrichs, Das Judenbild im Protestantismus des Deutschen Kaiserreichs. Ein Beitrag zur Mentalitätsgeschichte des deutschen Bürgertums in der Krise der Moderne (Kirchengeschichtliche Monografien, 12), Giessen 22004; Olaf Blaschke, Katholizismus und Antisemitismus im Deutschen Kaiserreich (Kritische Studien zur Geschichtswissenschaft, 122), Göttingen 1999. 
verschiebung von religiösen hin zu sozioökonomischen, kulturellen und nationalen Diskursen, die Kernelemente des sogenannten modernen Antisemitismus sind, der sich in jenen Jahren herausbildete. Religiöse Diskurse allerdings blieben weiterhin stark verbreitet. ${ }^{8}$

Die thematische Bezugnahme der ab Ende der 1870er-Jahre stark gehäuft auftretenden antisemitischen Artikel auf Deutschland war prädominant. ${ }^{9}$ Die Autoren stützten sich dabei oft auf Beiträge aus protestantischen Zeitschriften Deutschlands, wiederholt wurden gar große Teile der Artikel aus deutschen Druckerzeugnissen übernommen. ${ }^{10}$ Der Blick auf den deutschen Kontext schlug sich nicht nur in den umfangreichen antisemitischen Beiträgen, sondern auch in einer Vielzahl kürzerer Meldungen nieder, die in den Nachrichten-Rubriken der Zeitschriften Verbreitung fanden. So insbesondere im Appenzeller Sonntagsblatt, das in jener Zeit mit der außergewöhnlich hohen Auflage von rund 20.000 Stück als größtes schweizerisches Sonntagsblatt und auflagestärkste protestantische Zeitschrift der Schweiz galt. ${ }^{11}$

Eine herausragende Rolle als Katalysator für die Popularisierung des Antisemitismus im Deutschschweizer Protestantismus kam dabei der Rezeption der antisemitischen Agitation des Berliner Hofpredigers Adolf Stoecker zu, weswegen ausführlicher auf diesen Fall eingegangen werden soll. Als Leiter der Berliner Stadtmission war Stoecker zu einem international bekannten Prediger geworden, dessen Predigten auch im Deutschschweizer Protestantismus rege gelesen wurden. ${ }^{12}$ Die Rezeption setzte im Kontext seiner Aktivitäten in der

8 Ausführlich in meiner Dissertation: Thomas Metzger, Antisemitismus im Deutschschweizer Protestantismus 1870 bis 1950 (Studien zum Antisemitismus in Europa, 12), Berlin 2017.

9 Siehe als Beispiele für die Bezugnahme auf Deutschland: Ludwig Pestalozzi, Kirchliche Rundschau vom Dezember, in: Evangelisches Wochenblatt 21/2 (1880), S. 5-7, hier S. 6-7; Politisches, in: Christlicher Volksbote 48/47 (1880), S. 375-376, hier S. 376; Nachrichten und Korrespondenzen, in: Volksblatt für die reformirte Kirche der Schweiz 12/49 (1880), S. 195-196, hier S. 196; Rundschau zur Jahreswende, Teil II, in: Kirchenfreund 15/2 (1881), S. 17-23, hier S. 18; Ludwig Pestalozzi, Kirchliche Rundschau vom Januar, Teil II, in: Evangelisches Wochenblatt 22/6 (1881), S. 31-34, hier S. 32-33; [Johannes Friedrich Alexander de le Roi?], Die socialen Verhältnisse der Juden in Preußen, in: Freund Israels 12/4 (1885), S. 120-127; Politisches, in: Christlicher Volksbote 60/50 (1892), S. 399-400, hier S. 400.

10 Für die Bedeutung einzelner Zeitschriften als Medien des Antisemitismustransfers siehe weiter unten.

11 Verantwortlich für den Nachrichtenteil zeichnete der in Schaffhausen wirkende Pfarrer Johann Jakob Schenkel, der einen strikt antisemitischen Kurs fuhr. Siehe etwa: Johann Jakob Schenkel, Nachrichten, in: Appenzeller Sonntagsblatt 18/32 (1879), S. 254-256, hier S. 255; Derselbe, in: Appenzeller Sonntagsblatt 19/48 (1880), S. 382-384, hier S. 383-384; Derselbe, Nachrichten, in: Appenzeller Sonntagsblatt 20/36 (1881), S. 286-288, hier S. 287. Siehe zudem: Kirchliche Nachrichten, in: Kirchenfreund 12/23 (1878), S. 367; Nachrichten, in: Christlicher Volksbote 60/34 (1892), S. 270-272, hier S. 271.

12 Hans Engelmann, Kirche am Abgrund. Adolf Stoecker und seine antijüdische Bewegung 
,inneren Mission' ein und verstärkte sich ab der Gründung seiner ChristlichSozialen Arbeiterpartei im Jahr $1878 .{ }^{13}$ Mit Blick auf das theologisch-kirchenpolitische Richtungswesen schieden sich an ihm die Geister. Während Zeitschriften des konservativen Protestantismus den Hofprediger zum heroischen Vorkämpfer einer ,Rechristianisierung' stilisierten, brandmarkten die Organe des religiösen ,Freisinns' Stoecker als Exponenten der, Orthodoxie.${ }^{\text {c }}{ }^{14}$ Vor diesem Hintergrund erstaunt es nicht, dass auch die konsequente Inkorporierung des Antisemitismus in die politische Strategie Stoeckers ab 1879 auf Beachtung im Deutschschweizer Protestantismus stieß ${ }^{15}$ Die Wahrnehmung und Diskussion von Stoeckers Antisemitismus setzten unmittelbar nach dessen ersten beiden Judentum-Reden ${ }^{16}$ von September 1879 ein. Sie fanden im konservativen Pro-

(Studien zu jüdischem Volk und christlicher Gemeinde, 5), Berlin 1984, S. 152; Urs Hofmann, Adolf Stoecker in Basel. Antisemitismus und soziale Frage in der protestantischen Presse in den 1880er Jahren, unveröffentlichte Lizentiatsarbeit Universität Basel, 2003, S. 17. Betreffend die Lektüre der Predigten Stoeckers in der Schweiz: Conrad von Orelli, Adolf Stöcker †, in: Kirchenfreund 43/4 (1909), S. 53-58, hier S. 56.

13 Stoecker und sein Antisemitismus sind gut erforscht. Es sei exemplarisch auf folgende $\mathrm{Pu}$ blikationen verwiesen: Engelmann, Kirche am Abgrund (wie Anm. 12); Günter Brakelmann/ Martin Greschat/Werner Jochmann, Protestantismus und Politik. Werk und Wirkung Adolf Stoeckers (1835-1909) (Hamburger Beiträge zur Sozial- und Zeitgeschichte, 17), Hamburg 1982; Martin Greschat, Protestantischer Antisemitismus in Wilhelminischer Zeit. Das Beispiel des Hofpredigers Adolf Stoecker, in: Günter Brakelmann/Martin Rosowski (Hg.), Antisemitismus. Von religiöser Judenfeindschaft zur Rassenideologie, Göttingen 1989, S. 2751.

14 Für die konservative Perspektive etwa: Ludwig Pestalozzi, Kirchliche Rundschau, in: Evangelisches Wochenblatt 24/51 (1883), S. 227-228, hier S. 227; Politisches, in: Christlicher Volksbote 53/26 (1885), S. 208. Für die liberale Perspektive siehe z. B.: Christian Tester, Rundschau und Selbstschau, in: Religiöses Volksblatt 9/10 (1878), S. 76-80, hier S. 76; Im hehren deutschen Reich, in: Schweizerisches Protestantenblatt 5/4 (1882), S. 28-30, hier S. 28; Heinrich Frank, Chronik, in: Schweizerische Reformblätter 19/27 (1885), S. 215-216, hier S. 216.

15 Kern von Stoeckers Antisemitismus war ein konservativ-christlich geprägter integraler Nationalismus. Zugleich habe Stoecker, wie Martin Greschat betont, traditionelle Formen der Judenfeindschaft erweitert, modernisiert und transformiert: Greschat, Protestantischer Antisemitismus (wie Anm. 13), S. 34. Zum Antisemitismus Stoeckers siehe zudem: Heinrichs, Das Judenbild im Protestantismus (wie Anm. 7), passim; Ferrari Zumbini, Die Wurzeln des Bösen (wie Anm. 7), S. 151-165. Peter Pulzer deutet Stoeckers Politik als einen bewussten taktischen Entscheid: Peter G. J. Pulzer, Die Entstehung des politischen Antisemitismus in Deutschland und Österreich 1867-1914, Göttingen 2004, S. 138. Ähnlich auch: Werner Jochmann, Stoecker als nationalkonservativer Politiker und antisemitischer Agitator, in: Brakelmann/Greschat/Jochmann (Hg.), Protestantismus und Politik (wie Anm. 13), S. 145-148. Werner Bergmann hingegen betont, dass Stoeckers Antisemitismus nicht nur taktischem Kalkül entsprungen, sondern auch integraler Bestandteil und eine wesentliche Triebfeder seines Denkens und Handelns gewesen sei. Werner Bergmann, Art. Stoecker, Adolf, in: Wolfgang Benz (Hg.), Handbuch des Antisemitismus. Judenfeindschaft in Geschichte und Gegenwart, Bd. 2: Personen, Berlin 2009, S. 798-802, hier S. 799.

16 Adolf Stoecker, Unsere Forderungen an das moderne Judentum, in: Günter Brakelmann, Adolf Stoecker als Antisemit, Teil 2: Texte des Parteipolitikers und Kirchenmannes 
testantismus positive Aufnahme. Der pietistisch geprägte Christliche Volksbote aus Basel etwa druckte die Reden in großen Auszügen in zustimmendem Sinne ab. ${ }^{17}$ Die Strategie, Stoeckers Handeln als ,Notwehr ${ }^{6}$ zu rechtfertigen, und dessen antisemitisch motivierte Forderungen an das Judentum, ,ein wenig bescheidener“, „ein klein wenig toleranter“ zu sein und „etwas mehr Gleichheit“ walten zu lassen ${ }^{18}$, erhielten von Seiten der ,Positiven“ auch in den Folgejahren Zuspruch. Die drei Forderungen Stoeckers wurden fast zu geflügelten Begriffen. ${ }^{19}$ Auch spätere antisemitische Reden des politisierenden Hofpredigers stießen bei den ,Positiven' auf Beachtung. ${ }^{20}$ Die Berichterstattung über die antisemitischen Aktivitäten Stoeckers machte den Hauptteil der Rezeption des von 1879 bis 1881 dauernden Berliner Antisemitismusstreits im Deutschschweizer Protestantismus aus. Die antisemitischen Reden Stoeckers besaßen einen ausgeprägten Ereignischarakter, wobei mit Frank Bösch Ereignisse als verdichtete, Kommunikation auslösende Momente verstanden werden können, bei denen konkurrierende Erzählungen und Vorstellungen thematisch zentriert zusammenlaufen. ${ }^{21}$ Ereignisse erschüttern zudem Erfahrungsräume und wirken strukturverändernd. ${ }^{22}$ In diesem Sinne hat die Rezeption der antisemitischen Betätigung

(Schriften der Hans-Ehrenberg-Gesellschaft, 11), Waltrop 2004, S. 10-24; Adolf Stoecker, Notwehr gegen das moderne Judentum, in: Brakelmann, Adolf Stoecker als Antisemit (wie Anm. 16), S. 24-41.

17 Zeitfragen in Briefform. Ueber die Judenfrage, 4 Teile, in: Christlicher Volksbote, 47/44 (1879), S. 346-348; 47/45 (1879), S. 356-358; $47 / 46$ (1879), S. 364-366; 47/47 (1879), S. 370-372. Auch weitere konservative protestantische Periodika - insbesondere das Evangelische Wochenblatt aus Zürich - publizierten zustimmende Artikel: Ludwig Pestalozzi, Kirchliche Rundschau vom September, in: Evangelisches Wochenblatt 20/42 (1879), S. 190-192; Georg Rudolf Zimmermann, Das moderne Judenthum in Deutschland, besonders in Berlin. Zwei Reden von Adolf Stöcker, in: Evangelisches Wochenblatt 20/44 (1879), S. 199-201; Justus J. Heer, Rundschau auf die kirchlichen Zustände in Deutschland, besonders in Preußen, in: Kirchenfreund 13/24 (1879), S. 383-391, hier S. 385-386.

18 Stoecker, Unsere Forderungen an das moderne Judentum (wie Anm. 16), S. 14, S. 19, S. 22.

19 Siehe beispielsweise: Johann Jakob Schenkel, Nachrichten, in: Appenzeller Sonntagsblatt 23/ 46 (1884), S. 367-368, hier S. 368; Ludwig Pestalozzi, Zur Charakteristik A. Stöckers und seiner Bestrebungen, Teil III, in: Kirchenfreund 19/17 (1885), S. 257-261, hier S. 258.

20 Siehe beispielsweise: Politische Uebersicht, in: Allgemeine Schweizer Zeitung 6/243 (1879), S. 2; Johann Jakob Schenkel, Nachrichten, in: Appenzeller Sonntagsblatt 22/28 (1883), S. 223224; Jakob Schläpfer, Nachrichten, in: Appenzeller Sonntagsblatt 25/42 (1886), S. 334-336, hier S. 335 .

21 Frank Bösch, Ereignisse, Performanz und Medien in historischer Perspektive, in: Derselbe/ Patrick Schmidt (Hg.), Medialisierte Ereignisse. Performanz, Inszenierung und Medien seit dem 18. Jahrhundert, Frankfurt a. M./New York 2010, S. 7-29, hier S. 8.

22 Wie Andreas Suter und Manfred Hettling betonen, seien dies Eigenschaften, welche Ereignisse von ,normalem' Handlungsgeschehen unterscheiden würden: Andreas Suter/Manfred Hettling, Struktur und Ereignis - Wege zu einer Sozialgeschichte des Ereignisses, in: Dieselben (Hg.), Struktur und Ereignis (Geschichte und Gesellschaft: Sonderheft, 19), Göttingen 2001, S. 7-32, hier S. 23-25. 
Stoeckers im konservativen Protestantismus der Deutschschweiz in der Tat das Sprechen über , die Juden' verändert.

In den konservativ-protestantischen Zeitschriften, vor allem in jenen, die mit Stoeckers christlich-sozialen Vorstellungen sympathisierten, wurde der politisierende Hofprediger zu einem Heroen stilisiert. ${ }^{23}$ Im Kirchenfreund aus Basel etwa frohlockte mit Blick auf Stoeckers ,Judentum-Reden' der langjährige Redakteur, Pfarrer Justus J. Heer aus dem Kanton Zürich: „Gott wolle noch mehr Stöcker erwecken, welche in so ernster Weise die christlichen Gewissen aufwecken und unser Volk vor der Verjudung bewahren. “24

Insbesondere in den 1880er-Jahren hielt die starke Anteilnahme der ,Positiven', aber auch der, Vermittler' an den Aktivitäten des Hofpredigers an und mit ihr auch die Wiedergabe und Kommentierung seines Antisemitismus. Aus den Zeilen der konservativen Blätter sprachen Lob und Wertschätzung für seine Agitation. ${ }^{25}$ Ganz im Sinne der Konstruktion eines vermeintlich realen Konflikts - einer klassischen Argumentationsstrategie des Antisemitismus - wurde die Kampagne Stoeckers zudem als gerechtfertigt und als von ,den Juden' selbst provoziert dargestellt. ${ }^{26}$ Damit einhergehend wurde der Hofprediger, einer Verkehrung von Täter und Opfer folgend, zum eigentlichen Opfer stilisiert, das in ,Notwehr' gegen ,die Juden' gehandelt habe. ${ }^{27}$ Aufgrund der Aufmerksamkeit, die Stoeckers Ansichten in konservativ-protestantischen Kreisen der Deutschschweiz erhielten, erstaunt es nicht, dass es diesen zwischen 1881 und 1907 gelang, Stoecker für sechs Vortragsreisen $\mathrm{zu}$ gewinnen. ${ }^{28}$ Diese stießen auf

23 Siehe etwa: Justus J. Heer, Rundschau auf die kirchlichen Zustände in Deutschland, besonders in Preußen, in: Kirchenfreund 13/24 (1879), S. 383-391, hier S. 385; Johann Jakob Schenkel, Nachrichten, in: Appenzeller Sonntagsblatt 24/26 (1885), S. 207-208.

24 J. Justus Heer, Rundschau auf die kirchlichen Zustände in Deutschland, besonders in Preußen, in: Kirchenfreund 13/24 (1879), S. 383-391, hier S. 386.

25 Als Beispiele seien genannt: Carl Pestalozzi, Kirchliche Chronik, in: Christlicher Volksfreund 6/48 (1880), S. 424-426, hier S. 424; Ludwig Pestalozzi, Die Basler Festwoche, in: Evangelisches Wochenblatt 34/33 (1893), S. 139-142, hier S. 140. Für das Organ der, Vermittler' kann auf eine Artikelserie des Mitarbeiters und Stoecker-Bewunderers Pfarrer Johann Jakob Wälli verwiesen werden, der in dieser Zeitschrift mehrfach antisemitische Artikel verfasste: Jakob Wälli, Hofprediger Stöcker in Stuttgart, 2 Teile, in: Kirchenblatt für die reformierte Schweiz 3/24 (1888), S. 93-95; 3/25 (1888), S. 97-99.

26 Siehe u.a.: Nachrichten und Korrespondenzen, in: Volksblatt für die reformirte Kirche der Schweiz 12/49 (1880), S. 195-196; Politisches, in: Christlicher Volksbote 61/25 (1893), S. 199-200, hier S. 200.

27 Für diesen Diskurs siehe etwa: Ernst Miescher, Ein Mann des Volkes, Teil II, in: Christlicher Volksfreund 7/20 (1881), S. 177-180, hier S. 179-180; Jakob Wälli, Hofprediger Stöcker in Stuttgart, Teil I, in: Kirchenblatt für die reformierte Schweiz 3/24 (1888), S. 93-95, hier S. 93. Für den Topos der, Notwehr': Eine bedeutsame soziale Frage. Jüdisches, in: Volksblatt für die reformirte Kirche der Schweiz 9/48 (1879), S. 191; F., Die Nothwehr gegen die Juden, in: Evangelisches Wochenblatt 21/6 (1880), S. 24-25.

28 Stoecker besuchte auf seinen Vortragsreisen folgende Städte: 1881: Basel, Bern, Zürich; 1891: 
großes Interesse. Allerdings stellte Stoecker nie seine antisemitischen Ansichten ins Zentrum seiner Ausführungen, da er in der Schweiz vorab als religiöser Redner und Prediger, nicht aber als politischer Agitator auftrat. ${ }^{29}$ Die sehr positive Rezeption von Stoecker und dessen Antisemitismus dauerte konservativerseits in unterschiedlicher Intensität über dessen Tod im Jahre $1909^{30}$ hinaus fort. Ebenso wurde seine antisemitische Agitation retrospektiv verharmlost beziehungsweise begrüßt. ${ }^{31}$

Anders als der konservative Protestantismus reagierten die Liberalen sehr ablehnend auf Stoeckers politischen Protestantismus. Zugleich befassten sie sich weniger stark mit Stoeckers Ansichten. Sie assoziierten Stoecker mit der ihnen verhassten protestantischen, Orthodoxie' Preußens und sahen sich teilweise mit den Juden in einer gemeinsamen Opferrolle. ${ }^{32}$ So betonte Ende 1879 die in Bern erscheinende Zeitschrift Reform, dass nun den ,Orthodoxen' die Bekämpfung des ,freisinnigen ' Christentums nicht mehr ausreiche, nun müsse auch noch, der Jude dranglauben. ${ }^{33}$ Man war im Deutschschweizer Protestantismus für oder gegen den Antisemitismus Stoeckers, weil man grundsätzlich ein Anhänger oder Gegner des Hofpredigers war. Somit kritisierten die Liberalen Stoeckers Antisemitismus nicht etwa deshalb, weil sie die Judenfeindschaft per se für ver-

Basel, Bern, Genf; 1891 (auf der Rückreise von der Versammlung der Evangelischen Allianz in Florenz): Zürich; 1894: Basel, Olten, Bern; 1901: Basel; 1907: Basel. Da Stoecker bereits gesundheitlich angeschlagen war, sprach er auf seiner letzten Reise nur noch in privatem Rahmen. Die Referate Stoeckers wurden teilweise als Broschüren gedruckt. So etwa: Adolf Stoecker, Die soziale Frage im Lichte der Bibel. Vortrag gehalten Dienstag den 5. April 1881 in der französischen Kirche in Bern, Bern 1881.

29 Eine Ausnahme stellte sein Auftritt in Bern im Jahre 1881 dar, bei dem die,Judenfrage ' in der Diskussionsrunde thematisiert wurde. Dies wurde im Christlichen Volksboten aus Basel lobend erwähnt: Nachrichten, in: Christlicher Volksbote 49/15 (1881), S. 118-120, hier S. 119.

30 Anlässlich seines Todes erschienen teilweise umfangreiche Würdigungen. Siehe z.B.: Theodor Sarasin-Bischoff, Ein Blick in das Leben Hofprediger D. Ad. Stöckers, 3 Teile, in: Christlicher Volksbote 77/7 (1909), S. 50-52; 77/8 (1909), S. 60-61; 77/9 (1909), S. 69-70; Ludwig Pestalozzi, Adolf Stöcker, 2 Teile, in: Evangelisches Wochenblatt 50/7 (1909), S. 2527; 50/8 (1909), S. 29-31; Conrad von Orelli, Adolf Stöcker †, in: Kirchenfreund 43/4 (1909) 4, S. 53-58.

31 Siehe als Auswahl: Adolf Stöcker, 4 Teile, in: Christlicher Volksfreund 37/19 (1911), S. 208-211; 37/20 (1911), S. 220-225; 37/21 (1911), S. 233-237; 37/22 (1911), S. 245-247; E. St. [Ernst Staehelin?], Juden und Christen in Vergangenheit, Gegenwart und Zukunft, Teil VI, in: Christlicher Volksfreund 57/12 (1931), S. 138-141, hier S. 139.

32 Für die Ablehnung der,Orthodoxie‘ siehe: Gottfried Schönholzer, Eine andere Meinung, in: Religiöses Volksblatt 9/11 (1878), S. 83-85, Albert Kalthoff, Aus dem kirchlichen Leben Berlins, Teil I, in: Schweizerisches Protestantenblatt 7/41 (1884), S. 374-378, hier S. 377-378. Bezüglich der gemeinsamen Opferrolle beispielsweise: Salomon Zimmermann, Reformchronik, in: Reform 9/12 (1880), S. 197-200, hier S. 199; Die neueste Volksbeschwindelung, in: Schweizerisches Protestantenblatt 4/43 (1881), S. 352-353; Aus Staat und Kirche, in: Religiöses Volksblatt 21/46 (1890), S. 384-386.

33 Salomon Zimmermann, Reformchronik, in: Reform 8/25 (1879), S. 460-463, hier S. 460. 
werflich hielten, sondern weil sie seine dem Liberalismus diametral entgegen gerichteten theologischen, gesellschaftlichen und politischen Ansätze verabscheuten. Daher überrascht es auch nicht, dass sich in der liberalen Kritik von Stoeckers Antisemitismus ebenfalls judenfeindliche Motive manifestierten. ${ }^{34}$ Prototypisch zeigte Pfarrer Salomon Zimmermann im Dezember 1879 eine solche Verknüpfung von Aussagen gegen den Antisemitismus Stoeckers mit judenfeindlichen Bemerkungen:

„Wir möchten nun den gegen das moderne Judenthum erhobenen Klagen keineswegs alle Berechtigung absprechen. Es liegt etwas in dem allgemeinen Charakter dieses Volkes schon, das unmöglich unsere Sympathien erweckt, und der Schachergeist desselben, sein entsetzlicher Realismus, der sich so leicht über die Gewissenhaftigkeit wegsetzt und der schon so manches Bäuerlein und manchen Berufsmann ökonomisch zu Grunde gerichtet, ist nicht ohne Grund fast sprichwörtlich geworden. Aber in der Art und Weise, wie gegenwärtig der Krieg gegen die Juden geführt wird, liegt etwas Unwürdiges, etwas dem Geiste des Christenthums Widersprechendes. “ ${ }^{35}$

Letztlich war die theologisch-kirchenpolitische Richtung ausschlaggebend für die Haltung gegenüber Stoecker und dessen Antisemitismus. Die transnationale theologisch-weltanschauliche, konfessionelle, aber auch sprachlich-kulturelle Verbundenheit mit dem deutschen Protestantismus führte dazu, dass die ,Positiven' Stoeckers Antisemitismus breit rezipierten und wiedergaben. Daraus resultierte eine Verdichtung judenfeindlicher Diskurse, und Deutschland wurde für den konservativen Deutschschweizer Protestantismus noch ausgeprägter zum Referenzrahmen für antisemitische Haltungen, als er es bis anhin gewesen war.

\section{Transfer antisemitischer Diskurse in den Deutschschweizer Protestantismus}

Im Rahmen der durch eine stark germanophile und konfessionssolidarische Einstellung gesteuerten Rezeption des deutschen Antisemitismus kann ein Transfer antisemitischer Diskurse in den Deutschschweizer Protestantismus festgestellt werden. Judenfeindlichen Publikationen aus dem nördlichen Nachbarland kam dabei eine wichtige Rolle als Medien des Transfers zu. Auch diese

34 Dies war vor allem beim Schweizerischen Protestantenblatt aus Basel der Fall. Siehe z. B.: Alfred Altherr, Hofprediger Stöcker in Basel, in: Schweizerisches Protestantenblatt 4/14 (1881), S. 119-120, hier S. 119; Oskar Brändli, Kreuz und Quer, in: Schweizerisches Protestantenblatt 8/29 (1885), S. 266-267, hier S. 266; Aus der Rede gegen die Juden, 2 Teile, in: Schweizerisches Protestantenblatt 13/43 (1890), S. 341-342; 13/44 (1890), S. 350-354.

35 Salomon Zimmermann, Reformchronik, in: Reform, 8/25 (1879), S. 460-463, hier S. 461462. 
Dimension der Bedeutung des Kaiserreichs als transnationaler Referenzrahmen für den Antisemitismus im Deutschschweizer Protestantismus war fast ausschließlich auf die konservative und teilweise ,vermittlerische' Richtung beschränkt. Die im Zuge des Berliner Antisemitismusstreits stark zunehmende antisemitische Publizistik in Deutschland schlug sich in den Spalten der konservativ-protestantischen Zeitschriften nieder. So rezensierten diese einschlägige, deutsche Publikationen. Exemplarisch sei an dieser Stelle auf den Zürcher Theologieprofessor Conrad von Orelli verwiesen, der als Redakteur des Kirchenfreund im März 1880 lobend judenfeindliche Schriften des Historikers Heinrich von Treitschke, des Hofpredigers Adolf Stoecker sowie des Judenmissionars Johannes Friedrich Alexander de le Roi besprach. ${ }^{36}$

Eine weit wichtigere Rolle als Medien des Transfers spielten jedoch theologische und religiös geprägte politische Zeitschriften und Zeitungen aus Deutschland. Mit Blick auf die wichtige Funktion der Agitation Adolf Stoeckers als Katalysator für die Popularisierung des modernen Antisemitismus in den untersuchten, positiven' Periodika überrascht es nicht, dass dabei insbesondere christlich-soziale Presseerzeugnisse aus dem Umfeld des politisierenden Pastors von Bedeutung waren. Diese Periodika dienten als Artikellieferanten oder Nachrichtenquellen. Als Beispiele sind vorab der Reichsbote, aber auch die ebenfalls christlich-soziale Deutsche evangelische Kirchenzeitung zu nennen, wobei der als Organ des rechten Flügels der preußischen Konservativen fungierende Reichsbote das mit Abstand bedeutendste Transferorgan für antisemitische Aussagen darstellte. ${ }^{37}$ Insbesondere das in Basel gedruckte auflagenstarke Appenzeller Sonntagsblatt berief sich regelmäßig auf diese Zeitung. ${ }^{38}$ Auch

36 Conrad von Orelli, Literatur für und wider Israel, in: Kirchenfreund 14/6 (1880), S. 91-95, hier S. 91. Er bezog sich auf folgende Schriften: Heinrich von Treitschke, Ein Wort über unser Judenthum, Separatabdruck aus dem 44. und 45. Bande der Preußischen Jahrbücher, Berlin 1880; Adolf Stoecker, Das moderne Judenthum in Deutschland, besonders in Berlin. Zwei Reden in der christlich-socialen Arbeiterpartei, Berlin ${ }^{3} 1880$; Johannes Friedrich Alexander de le Roi, Stephan Schultz. Ein Beitrag zum Verständniß der Juden und ihrer Bedeutung für das Leben der Völker, Gotha ${ }^{2} 1878$.

37 Siehe zum Reichsboten: Dagmar Bussiek, Art. ,Der Reichsbote (1873-1936)‘, in: Wolfgang Benz (Hg.), Handbuch des Antisemitismus. Judenfeindschaft in Geschichte und Gegenwart, Bd. 6: Publikationen, Berlin/Boston 2013, S. 587-588; Dagmar Bussiek, „Das Gute gut und das Böse bös nennen“. Der Reichsbote 1873-1879, in: Michel Grunewald/Uwe Puschner (Hg.), Das evangelische Intellektuellenmilieu in Deutschland, seine Presse und seine Netzwerke (1871-1963), Bern 2008, S. 97-119. Für die Deutsche evangelische Kirchenzeitung, an der Stoecker selbst aktiv mitwirkte, siehe: Heinrichs, Judenbild des Protestantismus (wie Anm. 7), S. 213-250.

38 Aus der Vielzahl von antisemitischen Artikeln, die sich zu einem Großteil oder partiell auf den Reichsboten stützten, siehe als Auswahl: Johann Jakob Schenkel, Nachrichten, in: Appenzeller Sonntagsblatt 18/11 (1879), S. 86-87, hier S. 87; Derselbe, Nachrichten, in: Appenzeller Sonntagsblatt 20/24 (1881), S. 190-191; Derselbe, Nachrichten, in: Appenzeller Sonntagsblatt 21/38 (1882), S. 303-304, hier S. 303; Derselbe, Nachrichten, in: Appenzeller 
die im preußischen Konservatismus verankerte und wie die anderen beiden Blätter stark antisemitische Kreuzzeitung sowie die Neue Evangelische Kirchenzeitung fanden gelegentlich Beachtung. ${ }^{39}$

Die von den , positiven' Deutschschweizer Protestanten übernommenen oder ihnen als Informationsquelle dienenden antisemitischen Artikel aus dem deutschen Protestantismus befassten sich häufig mit der sogenannten ,Judenfrage'. Das antisemitische Konstrukt und die daran angegliederten judenfeindlichen Diskurse, die ,den Juden' beispielweise großen ,Einfluss' in Politik, Kultur, Wirtschaft und Medien unterstellten und ,die Juden' einer ,schädlichen', ja ,zersetzenden' Wirkung auf die Gesellschaft bezichtigten, erlebten unter den ,Positiven' im Kontext der Rezeption des Antisemitismusstreits ihren Durchbruch. Vor 1879 lässt sich der Terminus für die analysierten Zeitschriften nur äußerst selten nachweisen. ${ }^{40}$ Besonders populär war der Begriff im Appenzeller Sonntagsblatt, im Evangelischen Wochenblatt und in den pietistisch geprägten Zeitschriften Christlicher Volksbote und Freund Israels. ${ }^{41}$ Auf liberaler Seite fehlte der Begriff für diese Periode hingegen fast ganz. Die Ausnahme stellte das Schweizerische Protestantenblatt dar. ${ }^{42}$

Die Vorstellung, dass die Präsenz von Juden zu einer Frage respektive einem

Sonntagsblatt 22/37 (1882), S. 295-296, hier S. 295; Derselbe, Nachrichten, in: Appenzeller Sonntagsblatt 23/11 (1884), S. 87-88.

39 Zum Antisemitismus der Kreuzzeitung und der Neuen Evangelischen Kirchenzeitung: Dagmar Bussiek, „Mit Gott für König und Vaterland!“. Die Neue Preußische Zeitung (Kreuzzeitung) 1848-1892 (Schriftenreihe der Stipendiatinnen und Stipendiaten der FriedrichEbert-Stiftung, 15), Münster/Hamburg/London 2002; Heinrichs, Das Judenbild Protestantismus (wie Anm. 7), S. 156-213, v. a. S. 166-186; Engelmann, Kirche am Abgrund (wie Anm. 12), S. 51-61. Die Neue Evangelische Kirchenzeitung diente vorab in den 1870er-Jahren als Quelle für antisemitische Texte. Siehe z. B.: Politisches, in: Appenzeller Sonntagsblatt 10/51 (1871), S. 407-408; Politisches, in: Appenzeller Sonntagsblatt 11/41 (1872), S. 326-328, hier S. 327; Johann Jakob Schenkel, Nachrichten, in: Appenzeller Sonntagsblatt 12/35 (1873), S. 279-280, hier S. 280; Vermischtes, in: Evangelisches Wochenblatt 19/13 (1878), hier S. 58.

40 Sehr frühe Nennungen: Zur Judenfrage, in: Evangelisches Wochenblatt 1/10 (1860), S. 46-47; Zur Judenfrage, in: Evangelisches Wochenblatt 1/11 (1860), S. 52; Die Judenemanzipation im Aargau, in: Kirchenblatt für die reformirte Schweiz 19/11 (1863), S. 89-92.

41 Siehe als Auswahl aus den zahlreichen Artikeln: Johann Jakob Schenkel, Nachrichten, in: Appenzeller Sonntagsblatt 18/45 (1879), S. 358-360, hier S. 358-359; Ludwig Pestalozzi, Kirchliche Rundschau vom Dezember, in: Evangelisches Wochenblatt 21/2 (1880), S. 5-7; Friedrich Heman, Neunundvierzigster Jahresbericht der Freunde Israels zu Basel, in: Freund Israels 7/4 (1880), S. 81-98, hier S. 88; Johann Jakob Schenkel, Nachrichten, in: Appenzeller Sonntagsblatt 19/47 (1880), S. 374-376, hier S. 374; Ein Rückblick auf das Jahr 1880, Teil IV, in: Christlicher Volksbote 49/4 (1881), S. 29-31, hier S. 29.

42 Siehe etwa: Alfred Altherr, Die Judenfrage, in: Basler Protestantenblatt 2/35 (1879), S. 285290; Oskar Brändli, Kreuz und Quer, in: Schweizerisches Protestantenblatt 8/23 (1885), S. 211-213, hier S. 213. Siehe zudem folgende Artikelserie: Hans Emil Baiter, Die Judenfrage, 2 Teile, in: Schweizerisches Protestantenblatt 15/23 (1892), S. 180-181; 15/24 (1892), S. 188191. 
Problem geworden sei, war bis gegen Mitte der 1880er eine Schlüsselvorstellung im Antisemitismus der konservativ-protestantischen Zeitschriften. Der mit dem Konstrukt ,Judenfrage ' inhaltlich ebenfalls verknüpfte Topos der,Verjudung', in dem sich das antisemitische Bild einer vermeintlich zu großen jüdischen Präsenz noch akzentuierte, tauchte in den konservativen Texten gleichzeitig auf, doch war der Begriff deutlich weniger gebräuchlich als jener der ,Judenfrage ‘ ${ }^{43}$

Zentral für den Transfer des Topos der ,Judenfrage“ in den Deutschschweizer Protestantismus ist die Tatsache, dass eine direkte Übertragung des angeeigneten Topos auf den schweizerischen Kontext in den untersuchten Publikationen kaum je vollzogen wurde. ${ }^{44}$ Es ist bezeichnend und letztlich auch paradigmatisch für den Antisemitismus im Deutschschweizer Protestantismus im letzten Drittel des 19. Jahrhunderts, dass die antisemitischen Stellungnahmen am Ausland und in erster Linie an Deutschland abgehandelt wurden. Die ,Judenfrage' wurde somit exterritorialisiert und für Deutschland, nicht aber die Schweiz gestellt. ${ }^{45}$ Dasselbe ist von den eng mit dem Topos der ,Judenfrage' verknüpften antisemitischen Vorstellungen eines angeblich übermäßigen, jüdischen Einflusses` zu sagen. Dass ,die Juden' vorab in Deutschland eine ausgesprochene Machtsituation besitzen würden, war ein wiederkehrender antisemitischer Diskurs. ${ }^{46}$ So

43 Siehe beispielsweise: Johann Jakob Schenkel, Nachrichten, in: Appenzeller Sonntagsblatt 17/ 13 (1878), S. 102-104, hier S. 103; Mittheilungen über die Verhandlungen der evangelischen Allianz, Teil VI, in: Christlicher Volksbote 47/42 (1879), S. 330-333, hier S. 330; Israels Rückkehr nach Palästina, eine politische Zeitfrage oder religiöse Zukunftsfrage?, in: Freund Israels 5/2 (1878), S. 51-58, hier S. 56; Johann Jakob Schenkel, Nachrichten, in: Appenzeller Sonntagsblatt 27/21 (1888), S. 166-167, hier S. 167; Eduard Preiswerk, Festrede, in: Freund Israels 17/5 (1890), S. 121-129, hier S. 125.

44 Siehe als Ausnahme: Georg Rudolf Zimmermann, Das moderne Judenthum in Deutschland, besonders in Berlin. Zwei Reden von Adolf Stöcker, in: Evangelisches Wochenblatt 20/44 (1879), S. 199-201, hier S. 200. Die Entstehung einer ,Judenfrage' in der Schweiz erachteten auch als möglich: Rudolf Rüetschi, Miscelle. Zur Statistik der Juden, in: Volksblatt für die reformirte Kirche der Schweiz 12/33 (1880), S. 132; Rundschau zur Jahreswende, Teil II, in: Kirchenfreund 15/2 (1881), S. 17-23, hier S. 21.

45 Siehe beispielsweise: Eine bedeutsame soziale Frage. Jüdisches, in: Kirchenblatt für die reformirte Kirche der Schweiz 9/48 (1879), hier S. 191; Johann Jakob Schenkel, Nachrichten, in: Appenzeller Sonntagsblatt 20/10 (1881), S. 78-80, hier S. 89; Ludwig Pestalozzi, Kirchliche Rundschau vom Januar, Teil II, in: Evangelisches Wochenblatt 22/6 (1881), S. 31-34, hier S. 32-33; Johann Jakob Schenkel, Nachrichten, in: Appenzeller Sonntagsblatt 27/36 (1888), S. 285-287, hier S. 286.

46 Siehe als kleine Auswahl: Nachrichten und Correspondenzen, in: Volksblatt für die reformirte Kirche der Schweiz 4/37 (1872), S. 174-176, hier S. 175; Carl Pestalozzi, Kirchliche Chronik, in: Christlicher Volksfreund 3/34 (1877), S. 273-274, hier S. 273; Politisches, in: Christlicher Volksbote 45/38 (1877), S. 304; J. Alexander, Die gegenwärtige sociale und politische Lage des jüdischen Volkes, in: Freund Israels 5/1 (1878), S. 22-31, hier S. 27; Ludwig Pestalozzi, Kirchliche Rundschau vom Januar, Teil II, in: Evangelisches Wochenblatt 22/6 (1881), S. 31-34, hier S. 33; Johann Jakob Schenkel, Nachrichten, in: Appenzeller Sonntagsblatt 22/37 (1883), S. 295-296, hier S. 295. Das antisemitische Bild des ,jüdischen Einflusses' in Deutschland war vereinzelt auch auf liberaler Seite präsent. Siehe etwa: Salomon 
monierte etwa Pfarrer Carl Pestalozzi, Redakteur des Christlichen Volksfreunds, den angeblichen Zustand einer jüdischen ,Übermacht' in Deutschland und verwendete dabei die Symbolik der Söhne Noahs, die nach biblischem Verständnis als Stammväter für alle ,nachsintflutlichen Völker' angesehen wurden. In den Händen einer halben Million Juden würden sich, so der Zürcher Pfarrer, „ein so ungeheurer Reichthum und eine so grosse Macht“ vereinigen, dass „Japhet oft genug in den Hütten Sems wohnen und der Deutsche Haus und Feld von dem Israeliten zu lehen tragen " müsse. ${ }^{47}$

Die durch Solidarität mit theologischen Richtungen geprägte Positionsnahme für die antisemitischen Haltungen des deutschen konservativen Protestantismus war stark gekennzeichnet durch Rechtfertigungsdiskurse. Nicht zuletzt anhand statistischer Konstruktionen wurde wiederholt in typisch antisemitischer Manier ein Realkonflikt suggeriert, mit dem Ziel, die antisemitische Welle in Deutschland als gerechtfertigte und ,natürliche` Reaktion zu verharmlosen und den nördlichen Nachbarstaat in Schutz zu nehmen. ${ }^{48}$ Eine solche Argumentationsstrategie verfolgte beispielweise Pfarrer Johann Jakob Schenkel 1881 im konservativ-protestantischen Appenzeller Sonntagsblatt. Bezug auf Deutschland nehmend, reproduzierte er die Haltung des der Bewegung Stoeckers zuzurechnenden Reichsboten aus Berlin, wonach es kein Volk auf Dauer ertragen könne, „wenn die Juden in so großer Anzahl vorhanden“ seien, ${ }^{49}$ und 1884 fügte er im gleichen Sinne an, dass „eine aufstrebende Nation wie die deutsche [...] eine so große Uebermacht des Judenthums auf die Dauer" nicht ertrage. ${ }^{50}$

Mit Blick auf die Transfer-Dimension, die der Bedeutung des antisemitischen Diskurses im deutschen Protestantismus für die Judenfeindschaft im Protestantismus der Deutschschweiz zukam, gilt es abschließend nochmals festzuhalten: Die Rezeption der antisemitischen Welle in Deutschland machte das antisemitische Konstrukt der ,Judenfrage' vorab im konservativen Protestantismus der Deutschschweiz breit bekannt. Wie ist aber die Exterritorialisierung

Zimmermann, Reformchronik, in: Schweizerische Reformblätter 8/25 (1879), S. 460-463, hier S. 462.

47 Carl Pestalozzi, Kirchliche Chronik, in: Christlicher Volksfreund 6/48 (1880), S. 424-426, hier S. 424. Japhet wurde in dieser biblischen, Völkerkunde' als Urahne der europäischen Völker gesehen.

48 Siehe als Beispiele für die statistische Argumentationsstrategie: Zeitfragen in Briefform. Ueber die Judenfrage, Teil III, in: Christlicher Volksbote 47/46 (1879), S. 364-366, S. 365; Politisches, in: Christlicher Volksbote 48/47 (1880), hier S. 375; Nachrichten und Korrespondenzen, in: Volksblatt für die reformirte Kirche der Schweiz 17/22 (1885), hier S. 147148; Gottlieb Schuster, Kirchliche Zeitschau, in: Christlicher Volksfreund 15/32 (1889), 364-365, hier S. 365.

49 Johann Jakob Schenkel, Nachrichten, in: Appenzeller Sonntagsblatt 21/24 (1881), S. 190-191, hier S. 190.

50 Johann Jakob Schenkel, Nachrichten, in: Appenzeller Sonntagsblatt 23/46 (1884), S. 367-368, hier S. 368. 
der ,Judenfrage' mit Bezug auf den einem Transfer inhärenten Aspekt des Wandels respektive der Adaption eines übernommenen Konzepts zu deuten ? $^{51}$ Dieser Aspekt des Wandels kann darin gesehen werden, dass das dem Konstrukt der ,Judenfrage' zugrundeliegende antisemitische Denken bejaht, jedoch der imaginierte Realkonflikt in nationaler Hinsicht als ein nichtschweizerischer angesehen wurde.

\section{Deutschland als Kristallisationspunkt für antisemitische Aussagen}

Als dritte Dimension der transnationalen Bezugnahme auf die Entwicklungen in Deutschland kann abschließend deren Funktion als Kristallisationspunkt für die Artikulation fest etablierter antisemitischer Ansichten im Deutschschweizer Protestantismus verstanden werden. Das Interesse an den Vorgängen in Deutschland, das sich in den Spalten der untersuchten Zeitschriften niederschlug, legte antisemitische Einstellungen offen und ließ sie stärker manifest werden. Diese dritte Dimension ist eng verschränkt mit den beiden anderen Dimensionen. So zeigte sich das antisemitische Konzept ,Judenfrage' mit seiner gesellschaftsdeutenden Stoßrichtung offensichtlich als anschlussfähig für die etablierten Vorstellungen der ,Positiven'. Denn die Aneignung antisemitischer Diskurse ist, was auf Transferprozesse generell zutrifft, stark vom Rezipienten gesteuert. ${ }^{52}$ So war die Bezugnahme auf antisemitische Entwicklungen oder die Wiedergabe antisemitischer Texte das Ergebnis einer durch die Einstellungen des Rezipienten gesteuerten bewussten Selektion. Daher widerspiegelten etwa die zahlreichen im Nachrichtenteil des Appenzeller Sonntagsblattes abgedruckten Kurzberichte über das angebliche Fehlverhalten von Juden in Deutschland letztlich wohl auch das Bedürfnis, die eigenen antisemitischen Einstellungen zu bestätigen. Ein weiteres Beispiel dafür ist die in konservativprotestantischen Blättern wiederholt aufscheinende judenfeindliche Haltung, die den Aufstieg von Juden in gewisse gesellschaftliche Funktionen ablehnte. Der christliche Superioritätsanspruch stellte ein tragendes Element insbesondere des Antisemitismus der, Positiven' dar. Die rechtliche Gleichberechtigung wurde zwar nach der in der Schweiz 1866/74 erfolgten Emanzipation nicht mehr grundsätzlich in Frage gestellt, doch wurde sie nicht als ,gleiche Berechtigung

51 Die Bedeutung des Aspekts der Transformation in Transferprozessen betonen: Michel Espagne, Au delà du comparatisme, in: Derselbe, Les transferts culturels franco-allemands (Perspectives germaniques), Paris 1999, S. 35-49; Michael Werner/Bénédicte Zimmermann, Vergleich, Transfer, Verflechtung. Der Ansatz der Histoire croisée und die Herausforderung des Transnationalen, in: Geschichte und Gesellschaft 28 (2002), S. 607-636.

52 Zur Wichtigkeit der Auswahlmechanismen und Aneignungsstrategien in Rezeptionsprozessen: Werner, Zimmermann, Vergleich, Transfer, Verflechtung (wie Anm. 51), S. 613. 
für das Agieren in allen Bereichen der weiterhin als exklusiv-christlich gedachten Gesellschaft verstanden. ${ }^{53}$

Der christliche Superioritätsanspruch und damit einhergehend die Vorstellung, ,die Juden' hätten sich gesellschaftlich unterzuordnen, wurde insbesondere daran manifest, dass der Aufstieg von Juden in berufliche Positionen, die sie in ein hierarchisch übergeordnetes Verhältnis zu Christen stellte, abgelehnt wurde. Exemplarisch zeigte sich dies in den Feindbildern ,jüdischer Lehrer' und ,jüdischer Richter'. Auch hier war es wiederum paradigmatisch, dass die Zeitschriften oft auf Berichte aus Deutschland zurückgriffen, welche diese vorurteilsgesteuerte Wahrnehmung zu bestätigen schienen. ${ }^{54}$ Der Aufstieg von Juden in Autoritätspositionen deutete das Appenzeller Sonntagsblatt - eine ganze Fülle antisemitischer Stereotypen portierend - in endzeitlicher Weise als sogenannte Zeichen der Zeit. Vor allem mit Blick auf Deutschland folgerte es im Oktober 1880:

„Und das sollen wir Alles ruhig hinnehmen, sollen zusehen, wie diese Presse unser Volk religiös und damit auch sittlich ruinirt, es aber als Kulturfortschritt preisen, wenn Juden als Richter unserm Volk den Eid abnehmen und die Lehrer seiner Kindern in den Schulen sind, sollen es als Hebung des Nationalwohlstandes loben, wenn jüdische Bankiers jährlich Millionen verdienen, wenn jüdische Schacherer und Magazinhalter reiche Leute werden und in Städten Laden bei Laden und Haus bei Haus reihen, während unser deutsches christliches Volk verarmt?! ${ }^{\text {(55 }}$

Ähnlich gelagert war der Vorwurf, ,die Juden` würden den ,christlichen Sonntag‘ gefährden und den konservativen Kampf für einen arbeitsfreien, durch die Kirche strukturierten Sonntag hintertreiben. ${ }^{56}$

53 In der Schweiz wurde den Juden 1866 die Niederlassungs- und 1874 die Glaubens- und Kultusfreiheit gewährt.

54 Zum antisemitischen Feindbild des ,jüdischen Lehrers' siehe etwa: Johann Jakob Schenkel, Nachrichten, in: Appenzeller Sonntagsblatt 19/10 (1880), S. 78-80; hier S. 79; Conrad von Orelli, Literatur für und wider Israel, in: Kirchenfreund 14/6 (1880), S. 91-95, hier S. 94; Johann Jakob Schenkel, Nachrichten, in: Appenzeller Sonntagsblatt 21/21 (1882), S. 167-168, hier S. 168. Zu jenem des ,jüdischen Richters': Johann Jakob Schenkel, Nachrichten, in: Appenzeller Sonntagsblatt 20/5 (1881), S. 38-40, hier S. 39; Conrad von Orelli, Eine christliche Eidesverweigerung, in: Kirchenfreund 17/3 (1883), S. 37-41.

55 Zeichen der Zeit, in: Appenzeller Sonntagsblatt 19/41 (1880), S. 325-326, hier S. 326.

$56 \mathrm{Zu}$ den Vorwürfen gehörte etwa, Juden würden Christen dazu zwingen, am Sonntag zu arbeiten oder aber durch ihren angeblichen großen ,Einfluss' auf die Bevorzugung des jüdischen Sabbats gegenüber dem christlichen Sonntag hinwirken. Auch in dieser Argumentation wurde gerne mit Beispielen aus Deutschland argumentiert, welche diese vermeintlichen Machenschaften belegen würden. Siehe z. B.: Friedrich Heman, Rede am Epiphaniasfest 1882, in: Freund Israels 9/2 (1882), S. 25-33, S. 29; Gustav Peyer, Ein Kampf um den Sonntag, in: Schweizer Sonntagsfreund 3 (1883), S. 18-24, S. 19; Johann Jakob Schenkel, in: Appenzeller Sonntagsblatt 23/10 (1884), S. 151-152, hier S. 151; Johann Jakob Schenkel, Nachrichten, in: Appenzeller Sonntagsblatt 24/44 (1885), S. 351-352, hier S. 351; Gustav Peyer, Des Kaufmanns Sonntag, in: Schweizer Sonntagsfreund 21 (1886), S. 161-165, hier S. 162; H. L., 
Mit dem Aufkommen des sogenannten modernen Antisemitismus im Deutschschweizer Protestantismus vor dem Hintergrund der antisemitischen Welle in Deutschland ab Mitte der 1870er-Jahre verschwanden die Diskurse des religiös-theologisch argumentierenden Antijudaismus keineswegs. Das Beispiel dieser gesellschaftlichen Hierarchisierung aufgrund des christlichen Superioritätsanspruches zeigt auf, wie beide Diskussionsstränge sich miteinander verbinden konnten. Dies sei an dieser Stelle mit Blick auf die wichtige Frage des Ausmaßes an Kontinuität zwischen Antijudaismus und modernem Antisemitismus für die Antisemitismusforschung erwähnt. Gerade auch der Topos der Judenfrage' stellte eine weitere solche diskursive Brücke dar.

Der Antisemitismus im Deutschen Kaiserreich im Allgemeinen und im Protestantismus des nördlichen Nachbarn im Besonderen fungierte für den Deutschschweizer Protestantismus als transnationaler Bezugsrahmen. Seine Rezeption wirkte als ein Katalysator für die Popularisierung des modernen Antisemitismus ganz besonders im konservativen und, vermittlerischen Deutschschweizer Protestantismus. Damit einhergehend vollzog sich ein Transfer antisemitischer Konzepte, der sich beispielhaft an der Postulierung einer angeblich existierenden ,Judenfrage“ festmachen lässt. Da Rezeption und Übernahme selektive Prozesse sind, verweisen sie auf bereits etablierte Denkstrukturen. So wirkte Deutschland ebenso als Kristallisationspunkt für internalisierte antisemitische Vorstellungen. Indem auf die antisemitischen Entwicklungen im Kaiserreich Bezug genommen wurde, verfestigten sich diese judenfeindlichen Denkstrukturen weiter.

Das konfessionelle, sprachlich-kulturelle und oft auch theologisch-wissenschaftliche Verbundenheitsgefühl sowie die richtungsspezifische Solidarisierung mit dem Nachbarn im Norden blieben im Deutschschweizer Protestantismus bis zum Ersten Weltkrieg stark. Darin lag ein zentraler Grund für die fortdauernde Rezeption des Antisemitismus des deutschen Protestantismus. Der Ausgang des Ersten Weltkrieges schwächte die germanophile Einstellung unter den als theologische Bildungselite zu verstehenden Autoren und Redakteuren der untersuchten Zeitschriften ab, - eine Entwicklung, die letztlich auf die gesamte deutschschweizerische Bevölkerung zutraf. ${ }^{57}$ Weiterhin explizit germanophile Positionen vertretende Pfarrer neigten hingegen in der Folge richtungsübergreifend dazu, in ihrer Deutschland verteidigenden Haltung das Land als Opfer, der Juden' darzustellen. Diese Pfarrer wurden zu den zentralen Trägern von soziokulturell, national oder kulturalistisch-essentialisierend argumentie-

Vom Stuttgarter Sonntagscongreß, Teil IV, in: Christlicher Volksbote 60/24 (1892), hier S. 189-190, hier S. 190; Nachrichten, in: Christlicher Volksbote 60/34 (1892), S. 270-272, hier S. 271.

57 Für den Charakter der Deutschschweizer Germanophilie und deren Transformation siehe: Urner, Die Deutschen in der Schweiz (wie Anm. 2), v. a. S. 49-91. 
renden Diskursen des Antisemitismus im Deutschschweizer Protestantismus nach dem Ersten Weltkrieg. ${ }^{58}$ Sie zeigten sich auch für antisemitische Verschwörungstheorien empfänglich. ${ }^{59}$ Der Antisemitismus im Protestantismus Deutschlands stellte für diese Gruppe bis in die 1930er-Jahre einen wichtigen Referenzrahmen dar.

58 So trafen sich in ihrer antisemitischen Deutung der deutschen Situation nach dem Ersten Weltkrieg beispielsweise der ,positive` Wilhelm Hadorn und der liberale Hans Baur. Siehe beispielsweise: Wilhelm Hadorn, Die kirchliche Lage in Deutschland, in: Kirchenfreund 53/3 (1919), S. 22-23, hier S. 22; Hans Baur, Ich war hungrig, und ihr speistet mich. Eine Reise nach Oesterreich, Teil II, in: Schweizerisches Protestantenblatt 43/42 (1920), S. 333-335, hier S. 333-334; Wilhelm Hadorn, Chronik, in: Kirchenfreund 59/8 (1925), S. 123-126, hier S. 125. Richtungsübergreifend sympathisierten einige dieser Exponenten mit dem germanophilen Volksbund für die Unabhängigkeit der Schweiz, der aus der Agitation gegen den 1920 erfolgten Beitritt der Schweiz zum Völkerbund entstand und der in den 1930er-Jahren Berührungspunkte zum faschistischen Frontismus aufwies. Zum Volksbund siehe: Gilbert Grap, Differenzen in der Neutralität. Der Volksbund für die Unabhängigkeit der Schweiz (1921-1934), Zürich 2011.

59 Siehe z. B.: Hans Baur, Aus Sturm und Stille, in: Schweizerisches Protestantenblatt 44/39 (1921), S. 309; Derselbe, Aus Sturm und Stille, in: Schweizerisches Protestantenblatt 47/5 (1924), S. 38-39, hier S. 39. Besonders ausgeprägt waren diese in der jungreformierten Bewegung präsent, die extrem germanophile Positionen einnahm. Zu den Jungreformierten siehe: Aerne, Religiöse Sozialisten (wie Anm. 4), S. 38-48. 
Open-Access-Publikation im Sinne der CC-Lizenz BY 4.0

(c) 2019, V\&R unipress $\mathrm{GmbH}$, Göttingen 